\title{
Cardiovascular Protection with Danshensu in Spontaneously Hypertensive Rats
}

\author{
Yiqun TAng, ${ }^{*}$ Minhui Wang, Chunlin Chen, Xiaoyong Le, Shujuan Sun, and Yuemiao YIN \\ Research Division of Pharmacology, China Pharmaceutical University; No. 24 Tongjia Lane, Nanjing 210009, China. \\ Received April 25, 2011; accepted June 30, 2011
}

\begin{abstract}
The objective of the present study was to evaluate the cardiovascular protective effects of Danshensu, a water-soluble active component of Danshen, in spontaneously hypertensive rats (SHR). SHR (male, 9 weeks old, $n=30)$ were divided into three groups: 1$)$ saline control $(n=10) ; 2)$ a Danshensu $(10 \mathrm{mg} / \mathrm{kg} / \mathrm{d}$, intraperitoneally (i.p.)) treatment group $(n=10)$; and 3) a Valsartan $(10 \mathrm{mg} / \mathrm{kg} / \mathrm{d}$, intragastrically (i.g.)) treatment group $(n=10)$. Age-matched Wistar-Kyoto rats $(n=10)$ were used as normotensive controls. Saline and drug treatments were administered for 6 weeks. When the rats were 15 weeks old, their hearts were excised and arrhythmias were induced by an ex vivo ischemia/reperfusion protocol. The heart weight to body weight index was significantly increased in SHR, and this increase was attenuated with Danshensu treatment (both $p<0.05$ ). Systolic blood pressure and diastolic blood pressure were also decreased with Danshensu treatment, from $145 \pm 3$ and $103 \pm 10 \mathrm{mmHg}$ to $116 \pm 7$ and $87 \pm 2 \mathrm{mmHg}$ in SHR and Danshensu-treated groups, respectively (both $p<0.05$ ). The incidences of ventricular tachycardia and ventricular fibrillation decreased from 100 to $50 \%$ and $30 \%$ in SHR, respectively, with Danshensu treatment (both $p<0.05$ ). Serum nitric oxide content and inducible nitric oxide synthase activity were significantly increased with Danshensu (both $p<0.05$ ). In addition, Danshensu increased the $\mathrm{K}^{+}$current density and $\mathrm{Ca}^{2+}$ activated $\mathrm{K}^{+}$channel current density of mesenteric vascular smooth muscle cells isolated from SHRs. Together, these results demonstrate that Danshensu imparts cardiovascular protection by modifying vascular responses during the progression of hypertension.
\end{abstract}

Key words Danshensu; hypertrophy; spontaneously hypertensive rat; arrhythmic; $\mathrm{Ca}^{+}$-activated $\mathrm{K}^{+}$channels

Cardiovascular disease is a leading cause of death, and hypertension is a critical risk factor for cardiovascular events. The pathogenesis of hypertension is accompanied by decreased nitric oxide (NO) bioavailability in the vasculature and increased cardiovascular remodeling. ${ }^{1,2)}$ Hypertensive patients frequently develop clinically evident cardiac hypertrophy 10 to 20 years after the onset of hypertension, as a result of adaptive and maladaptive responses to pressure overload. Cardiac hypertrophy has been linked to the development of a variety of cardiovascular diseases, including myocardial ischemia, arrhythmias, and sudden cardiac death. Therefore, treatment options that not only maintain stable pressure levels but also delay or even regress the structural and functional changes in resistance arteries and the heart are needed. Despite the current availability of multiple anti-hypertensive medication types, a significant number of patients do not respond to treatment and remain hypertensive. As multiple mechanisms likely contribute to the development of hypertension, including angiotensin, oxidative stress and hemodynamic changes, multi-targeted therapeutic interventions will likely be required for effective management of hypertension.

Danshen is the dried root of the traditional Chinese herb Salvia miltiorrhiza that belongs to the family of Labiatae. Danshen is a popular traditional Chinese medicine and has been widely used in both Asian and western countries for the treatment of various diseases, including hepatitis, cerebrovascular diseases, and cardiovascular diseases such as angina pectoris, myocardial infarction and stroke. ${ }^{3,4)}$ Danshensu, chemical name: 3-(3,4-dihydroxyphenyl)-2-hydroxy-propanoic acid, is the major water-soluble component of Danshen (Fig. 1). Cardiotonic pills, extracted from Salvia miltiorrhiza, Panax notoginseng and Borneol, are effective in pro- tecting against ischemia-reperfusion induced microcirculatory disturbances and myocardial damage in the rat when used at doses of 100,400 , or $\left.800 \mathrm{mg} / \mathrm{kg}^{5}{ }^{5}\right)$ Danshensu has been shown to exert several pharmacologic activities, including anti-oxidative and hydroxyl radical scavenging roles. ${ }^{6}$ Our own previous data has shown that $10 \mathrm{mg} / \mathrm{kg}$ Danshensu pretreatment can: 1) effectively inhibit ischemia/reperfusioninduced arrhythmias in hearts with hypertrophy induced by Levothyroxine; 2) prevent hypertrophy progression, normalize serum nitric oxide (NO) content and endothelial nitric oxide synthase (eNOS) activity ${ }^{7}$; and 3) improve the function of the thoracic aorta by enhancing vasodilator sensitivity and depressing vasoconstrictor sensitivity. ${ }^{8}$ In cardiomyopathy induced by isoproterenol, $10 \mathrm{mg} / \mathrm{kg}$ Danshensu showed significant cardioprotective effects by reducing free radical levels, infarct size, and cardiac remodeling. ${ }^{9)}$

In the present study, we evaluated the cardiovascular protective potential of chronic treatment with Danshensu in spontaneously hypertensive rats (SHR). Large conductance $\mathrm{Ca}^{2+}$-activated $\mathrm{K}^{+}$(BKCa) channels, formed by an ion-conducting $\alpha$ subunit and a regulatory $\beta_{1}$ subunit, ${ }^{10)}$ are considered crucial for regulating vascular tone and blood pressure. $\mathrm{BKCa}$ channels have been suggested as novel potential drug targets in hypertension. ${ }^{11,12)}$ We used tetraethylammonium (TEA) as a selective BKCa channel inhibitor. ${ }^{13,14}$ We are the first to report that Danshensu chronic administration can re-<smiles>O=C(O)C(O)Cc1ccc(O)c(O)c1</smiles>

Fig. 1. Structure of Danshensu 
verse $\mathrm{K}^{+}$and $\mathrm{BKCa}$ currents down-regulation in SHR mesenteric arteries. Danshensu activates BKCa channels and stimulates the $\mathrm{NO} /$ cyclic guanosine 5'-monophosphate (NO/cGMP) pathway to play a role in its cardiovascular protective effect. We used Valsartan, an angiotensin II receptor blocker, as a positive reference drug to compare the protective effects of Danshensu.

\section{MATERIALS AND METHODS}

Drug and Chemicals Danshensu, $>98 \%$ pure, was purchased from Nanjing Zelang Pharmaceutical Technology Co., Ltd.; and Valsartan was purchased from Beijing Novartis Pharmaceutical Co., Ltd. (Batch No. x0395). NO, total nitric oxide synthase (NOS) and inducible nitric oxide synthase (iNOS) kits were purchased from Nanjing Jiancheng Institute of Biological Engineering. Collagenase I and bovine serum albumin (BSA) were obtained from Nanjing Sunshine Biotechnology Ltd., while papain was supplied by Sangon Bioengineering Institute (Shanghai, China). Tetraethylammonium was purchased from Sigma Chemical Co. (U.S.A.).

Animals All animal experiments were performed in accordance with the Guidelines for the Care and Use of Laboratory Animals published by the U.S. National Institutes of Health and conformed to the Animal Regulations of Jiangsu Province. All experiments were performed with the approval of the President of China Pharmaceutical University. SHR (9 weeks old) and age-matched Wistar-Kyoto rats (WKY), male, weighing $250 \pm 20 \mathrm{~g}$, were purchased from Shanghai Slac Laboratory Animal Co., Ltd. (Shanghai, China). Rats were kept in a room temperature controlled room $\left(25^{\circ} \mathrm{C}\right)$, with $12 \mathrm{~h}$ dark and $12 \mathrm{~h}$ artificial illumination daily (7:0019:00). Food and water were available ad libitum.

Animal Groups Thirty SHRs were randomly divided into three groups of 10 animals each. The control SHR group received saline $(0.9 \%)$ orally for 6 weeks. The Danshensu group received daily injections of Danshensu $(10 \mathrm{mg} / \mathrm{kg} / \mathrm{d}$, intraperitoneally (i.p.)), as previously described, for 6 weeks. The Valsartan treatment group received Valsartan $(10 \mathrm{mg} / \mathrm{kg} / \mathrm{d})$ orally for 6 weeks. The WKY normotensive control rats $(n=10)$ received saline $(0.9 \%)$ orally for 6 weeks. For each of the downstream analyses, at least 6 animals in each group were used.

Electrocardiograms Parameters, Blood Pressure, Arrhythmia Scores, Malignant Arrhythmia Incidence and Heart Weight to Body Weight Index Measurements After the 6-week treatment period, the rats were anesthetized with urethane $(1.0 \mathrm{~g} / \mathrm{kg}$, i.p.) while lead II electrocardiograms (ECG) were continuously recorded. Two polyethylene tubes (PE 50) filled with heparinized saline were catheterized into the abdominal aorta. The catheter in the abdominal aorta was connected, via a pressure transducer, to a biological function lab recording system BL-420F (Chengdu, China) for in-line measurement of blood pressure. During this period, the rats were placed on a warm bed kept at $37^{\circ} \mathrm{C}$.

After the blood pressure measurements, the hearts were rapidly excised, placed in ice-cold perfusion buffer, cannulated via the aorta and perfused in the Langendorff apparatus. The perfusion flow was maintained at a constant rate of $12 \mathrm{ml} \cdot \mathrm{min}^{-1}$ and with perfusate warmed to $37 \pm 1^{\circ} \mathrm{C}$. The perfusion solution was standard Krebs-Henseleit buffer
$(\mathrm{K}-\mathrm{H})$ gassed with $100 \% \mathrm{O}_{2}$ containing (in mmol/1; $\mathrm{pH} 7.4$ ): $\mathrm{NaCl} 118.5, \mathrm{KCl} 4.70, \mathrm{CaCl}_{2} 1.80, \mathrm{MgSO}_{4} 1.20, \mathrm{~N}$-(2-hydroxyethyl)piperazine- $N^{\prime}$-2-ethanesulfonic acid (HEPES) 20, $\mathrm{KH}_{2} \mathrm{PO}_{4} 1.20$, and glucose 11.10 . After $30 \mathrm{~min}$ of stabilization, the left descending coronary artery was ligated for $20 \mathrm{~min}$, followed by $30 \mathrm{~min}$ reperfusion. ECG was recorded by the surface of the heart, with one electrode in contact with the cardiac apex and another electrode in contact with the upper left ventricle at the root of the coronary artery. Arrhythmia scores were calculated in accordance with the Lambeth Conference standards: 0 points: no arrhythmia; 1 point: occasion ventricular premature beats (VPBs); 2 points: frequent VPBs (bigeminal rhythm or trigeminy); 3 points: short runs of ventricular tachycardia (VT); 4 points: sustained VT; 5 points: ventricular flutter; 6 points: ventricular fibrillation (VF); and 7 points: ventricular fibrillation to death. We focused on the measurements of the first occurrence time of VT, VF over the whole period of ischemia-reperfusion, and durations of VT and VF.

The hearts were taken down from the Langendorff apparatus and blotted with filter paper. The pericardium and large vessels were removed, and the atrium, right ventricle, and left ventricles were separated and weighed individually. The heart weight to body weight $(\mathrm{HW} / \mathrm{BW})$ and left ventricular weight to body weight (LVW/BW) were calculated. The sections were dried in an oven $\left(50^{\circ} \mathrm{C}\right)$ for $24 \mathrm{~h}$ and dry weights were obtained. Myocardial water content (MWC) was calculated as the wet weight of the heart subtracted by the dry weight of the heart divided by the wet weight of the heart.

Determination of NO Content and NOS Activity in Serum Blood was sampled from the abdominal aorta, and serum was obtained after centrifugation at $3000 \mathrm{rpm} / \mathrm{min}$ for $10 \mathrm{~min}$. Serum NO levels were determined by measuring the concentration of nitrite from nitrates, a stable metabolite of NO, by the modified Griess reaction method. The total NOS and iNOS activities were determined according to the manufacturer's instructions.

Isolation of Mesenteric Vascular Smooth Muscle Cells The viscera of rats were exposed, and a proximal segment of the small bowel was removed and pinned in Tyrode's solution of the following composition (in mmol/l): $\mathrm{NaCl} 137 ; \mathrm{KCl}$ 5.4; $\mathrm{MgCl}_{2}$ 1.2; $\mathrm{CaCl}_{2}$ 1.2; taurine 10; HEPES 10; and glucose 10 . The $\mathrm{pH}$ was adjusted to 7.4 with $\mathrm{NaOH}$. Branch II or III resistance arteries were cleaned of fat and connective tissue, and a segment 1.6 to $2.0 \mathrm{~mm}$ in length was collected and placed in $\mathrm{Ca}^{2+}$-free Tyrode's solution containing $0.5 \mathrm{mg} / \mathrm{ml}$ papain and $1 \mathrm{mg} / \mathrm{ml}$ dithiothreitol (DTT) to incubate at $37^{\circ} \mathrm{C}$ for $20 \mathrm{~min}$. The supernatant was removed, and the segment was incubated in Tyrode's solution containing $1 \mathrm{mg} / \mathrm{ml}$ collagenase I, $2 \mathrm{mg} / \mathrm{ml} \mathrm{BSA}$ and $0.5 \mathrm{mmol} / 1 \mathrm{CaCl}_{2}$ at $37^{\circ} \mathrm{C}$ for $15 \mathrm{~min}$. Cells were stored in extracellular solution and used within $8 \mathrm{~h}$ of isolation.

Whole-Cell Patch-Clamp Technique Membrane currents were measured using the whole-cell configuration of the patch-clamp technique in voltage-clamped mesenteric vascular smooth muscle cells from SHRs, WKY, Danshensutreated and Valsartan-treated groups. Recording pipettes, made from borosilicate glass $(1.2 \mathrm{~mm}$, o.d.), pulled with a pipette puller (PIP5, HEKA, Germany), had resistances of 4 to $6 \mathrm{M} \Omega$ when filled with the internal solution. The internal solution was (in mmol/l): $\mathrm{KCl} 20$; $\mathrm{K}$-aspartate; $\mathrm{KH}_{2} \mathrm{PO}_{4} 1$; 
Table 1. Effect of Danshensu on ECG Properties

\begin{tabular}{lrcrr}
\hline \hline \multicolumn{1}{c}{ Group } & WKY & SHR & Valsartan & Danshensu \\
\hline HR (beat/min) & $297 \pm 54$ & $346 \pm 28^{\#}$ & $292 \pm 37^{*}$ & $283 \pm 23^{*}$ \\
P wave (ms) & $56 \pm 28$ & $86 \pm 10^{\#}$ & $64 \pm 8^{*}$ & $69 \pm 13^{*}$ \\
QT interval (ms) & $85 \pm 36$ & $121 \pm 5^{\#}$ & $90 \pm 38^{*}$ & $94 \pm 24^{*}$ \\
QTc (ms) & $207 \pm 36$ & $290 \pm 7^{\#}$ & $194 \pm 70^{*}$ & $205 \pm 51^{*}$ \\
QRS duration (ms) & $52 \pm 17$ & $82 \pm 13^{\#}$ & $54 \pm 18^{*}$ & $56 \pm 25^{*}$
\end{tabular}

Values are presented as mean \pm S.D.; $n=10$ in each group. The doses of Valsartan and Danshensu were both $10 \mathrm{mg} / \mathrm{kg}$ per day for 6 weeks. $\# p<0.05 v s$. WKY. $* p<0.05 v s$. SHR.
Table 2. Effect of Danshensu on Blood Pressure and Heart Weight Index

\begin{tabular}{lclcc}
\hline \hline \multicolumn{1}{c}{ Group } & WKY & SHR & Valsartan & Danshensu \\
\hline SBP (mmHg) & $95 \pm 8$ & $145 \pm 3^{\#}$ & $108 \pm 5^{*}$ & $116 \pm 7 *$ \\
DBP (mmHg) & $61 \pm 4$ & $103 \pm 10^{\#}$ & $83 \pm 10^{*}$ & $87 \pm 2 *$ \\
HW/BW $(\mathrm{g} / \mathrm{kg})$ & $4.35 \pm 0.46$ & $5.24 \pm 0.56^{\#}$ & $4.90 \pm 0.05^{*}$ & $4.80 \pm 0.40^{*}$ \\
LVW/BW $(\mathrm{g} / \mathrm{kg})$ & $0.97 \pm 0.16$ & $1.42 \pm 0.35^{\#}$ & $1.09 \pm 0.10^{*}$ & $1.07 \pm 0.13^{*}$ \\
MWC (\%) & $77.6 \pm 4.37$ & $71.1 \pm 5.99^{\#}$ & $78.7 \pm 1.64 *$ & $77.7 \pm 2.49^{*}$
\end{tabular}

Values are presented as mean \pm S.D.; $n=10$ in each group. The doses of Valsartan and Danshensu were both $10 \mathrm{mg} / \mathrm{kg}$ per day for 6 weeks. $\# p<0.05 v s$. WKY. $* p<0.05 v s$. SHR.

Table 3. Effect of Danshensu on VT and VF Incidence, Onset Times and Duration

\begin{tabular}{|c|c|c|c|c|c|c|}
\hline \multirow{2}{*}{ Group } & \multicolumn{3}{|c|}{ Tachycardia } & \multicolumn{3}{|c|}{ Fibrillation } \\
\hline & Incidence $(\%)$ & Onset(s) & Duration(s) & Incidence $(\%)$ & Onset(s) & Duration(s) \\
\hline WKY & 40 & $84.2 \pm 18.3$ & $245.0 \pm 24.3$ & 30 & $266.0 \pm 22.6$ & $158.6 \pm 20.0$ \\
\hline SHR & $100^{\#}$ & $59.2 \pm 29.0^{\#}$ & $401.4 \pm 17.2^{\#}$ & $100^{\#}$ & $142.0 \pm 25.9^{\#}$ & $340.9 \pm 20.8^{\#}$ \\
\hline Valsartan & $60 *$ & $75.6 \pm 14.9 *$ & $247.5 \pm 15.8^{*}$ & $50 *$ & $206.0 \pm 22.0 *$ & $187.0 \pm 17.1^{*}$ \\
\hline Danshensu & $50 *$ & $73.3 \pm 12.4 *$ & $253.5 \pm 23.8^{*}$ & $30 *$ & $181.5 \pm 22.4 *$ & $181.0 \pm 15.8^{*}$ \\
\hline
\end{tabular}
SHR.

Values are presented as mean \pm S.D.; $n=10$ in each group. The doses of Valsartan and Danshensu were both $10 \mathrm{mg} / \mathrm{kg}$ per day for 6 weeks. $\# p<0.05 v s$. WKY. $* p<0.05 v s$.

Table 4. Effect of Danshensu on Cardiac Arrhythmia Scores in Response to Ischemia-Reperfusion

\begin{tabular}{|c|c|c|c|c|c|c|c|c|c|}
\hline \multirow{2}{*}{ Group } & \multirow{2}{*}{ Pre } & \multicolumn{3}{|c|}{ Ischemia period (min) } & \multicolumn{5}{|c|}{ Reperfusion period (min) } \\
\hline & & 5 & 10 & 15 & 1 & 5 & 10 & 20 & 30 \\
\hline WKY & $0.0 \pm 0.0$ & $2.2 \pm 1.0$ & $3.3 \pm 0.6$ & $4.8 \pm 0.6$ & $3.3 \pm 0.6$ & $5.3 \pm 0.6$ & $5.3 \pm 0.0$ & $4.7 \pm 1.5$ & $3.3 \pm 0.6$ \\
\hline SHR & $3.0 \pm 0.9^{\#}$ & $4.6 \pm 0.6^{\#}$ & $5.7 \pm 0.7^{\#}$ & $5.8 \pm 0.7^{\#}$ & $4.1 \pm 1.0^{\#}$ & $6.5 \pm 0.9^{\#}$ & $5.9 \pm 0.6^{\#}$ & $5.9 \pm 0.9^{\#}$ & $7.0 \pm 0.0^{\#}$ \\
\hline Valsartan & $1.75 \pm 0.5^{*}$ & $2.0 \pm 0.8^{*}$ & $3.25 \pm 0.5^{*}$ & $5.25 \pm 0.5^{*}$ & $4.0 \pm 0.8$ & $5.5 \pm 0.6^{*}$ & $5.2 \pm 0.5^{*}$ & $4.3 \pm 1.0 *$ & $3.0 \pm 0.9^{*}$ \\
\hline Danshensu & $1.5 \pm 0.6^{*}$ & $2.0 \pm 0.8 *$ & $3.25 \pm 1.0 *$ & $5.0 \pm 0.8 *$ & $3.8 \pm 1.0$ & $5.0 \pm 0.8^{*}$ & $5.2 \pm 1.0 *$ & $4.7 \pm 0.5^{*}$ & $4.5 \pm 2.1 *$ \\
\hline
\end{tabular}
SHR.

Values were presented as mean \pm S.D.; $n=10$ in each group. The doses of Valsartan and Danshensu were both $10 \mathrm{mg} / \mathrm{kg}$ per day for 6 weeks. $\# p<0.05 v s$. WKY. $* p<0.05 v s$.

$\mathrm{MgCl}_{2} 1$; ethylene glycol bis(2-aminoethylether)- $N, N, N^{\prime}, N^{\prime}-$ tetraacetic acid (EGTA) 0.1; $\mathrm{Na}_{2}$ ATP 3; HEPES 5. The $\mathrm{pH}$ was adjusted to 7.2 with $\mathrm{KOH}$. Tip potentials were compensated before the pipette touched the cell. The voltage-clamp protocol to generate BKCa currents was as follows: the holding potential was $-40 \mathrm{mV}$ and a range of voltage steps from -40 to $+60 \mathrm{mV}$ for $500 \mathrm{~ms}$ to induce outward potassium currents. TEA ( $2 \mathrm{mmol} / \mathrm{l})$ was added to inhibit BKCa current. All experiments were performed at $25^{\circ} \mathrm{C}$.

Statistical Analysis All data were expressed as mean \pm S.D. For normally distributed data, Student's $t$-test was used to determine the statistical differences between two groups. Intergroup differences for three or more groups were analyzed by one-way analysis of variance (ANOVA). If one or more of the groups were not normally distributed, nonparametric statistics were used. A value of $p<0.05$ was considered as statistically significant.

\section{RESULTS}

Effects of Danshensu on ECG Parameters and Blood Pressure Compared with WKY normotensive rats, the QRS wave of SHR rats was widened, and the QTc was significantly prolonged (both $p<0.05$ ). These electrocardiographic changes were attenuated in SHR rats treated with
Danshensu or Valsartan (Table 1). Compared to the SHR control group, rats treated with either Danshensu or Valsartan showed significantly reduced systolic (SBP) and diastolic blood pressures (DBP) (all $p<0.05)$. While SHR blood pressures were significantly elevated compared to the WKY normotensive controls, Danshensu and Valsartan both prevented the increase in blood pressure (Table 2).

Effects of Danshensu on Arrhythmias and Heart Weight Index VT and VF incidences, onset times, and duration times are shown in Table 3. In the WKY group, I/R induced a $40 \%$ VT incidence and a $30 \%$ VF incidence. Incidences of both significantly increased to $100 \%$ in the SHR group. VT and VF incidences were significantly reduced in the Danshensu treated group to $50 \%$ and $30 \%$, respectively. VT and VF incidences were also significantly reduced in the Valsartan treated group to $60 \%$ and $50 \%$, respectively. The SHR group also showed increased VT and VF lasting durations and shortened onset times, compared to the WKY controls. Danshensu and Valsartan treatment delayed VT and VF onset times and shortened the duration times (all $p<0.05$ ). In the SHR group, cardiac arrhythmia scores increased compared to the WKY group $(p<0.05)$. Both Danshensu and Valsartan treatments reduced arrhythmia scores compared to the untreated SHR group (Table 4). Compared to the SHR groups, the $\mathrm{HW} / \mathrm{BW}$ and $\mathrm{LVW} / \mathrm{BW}$ indices were signifi- 

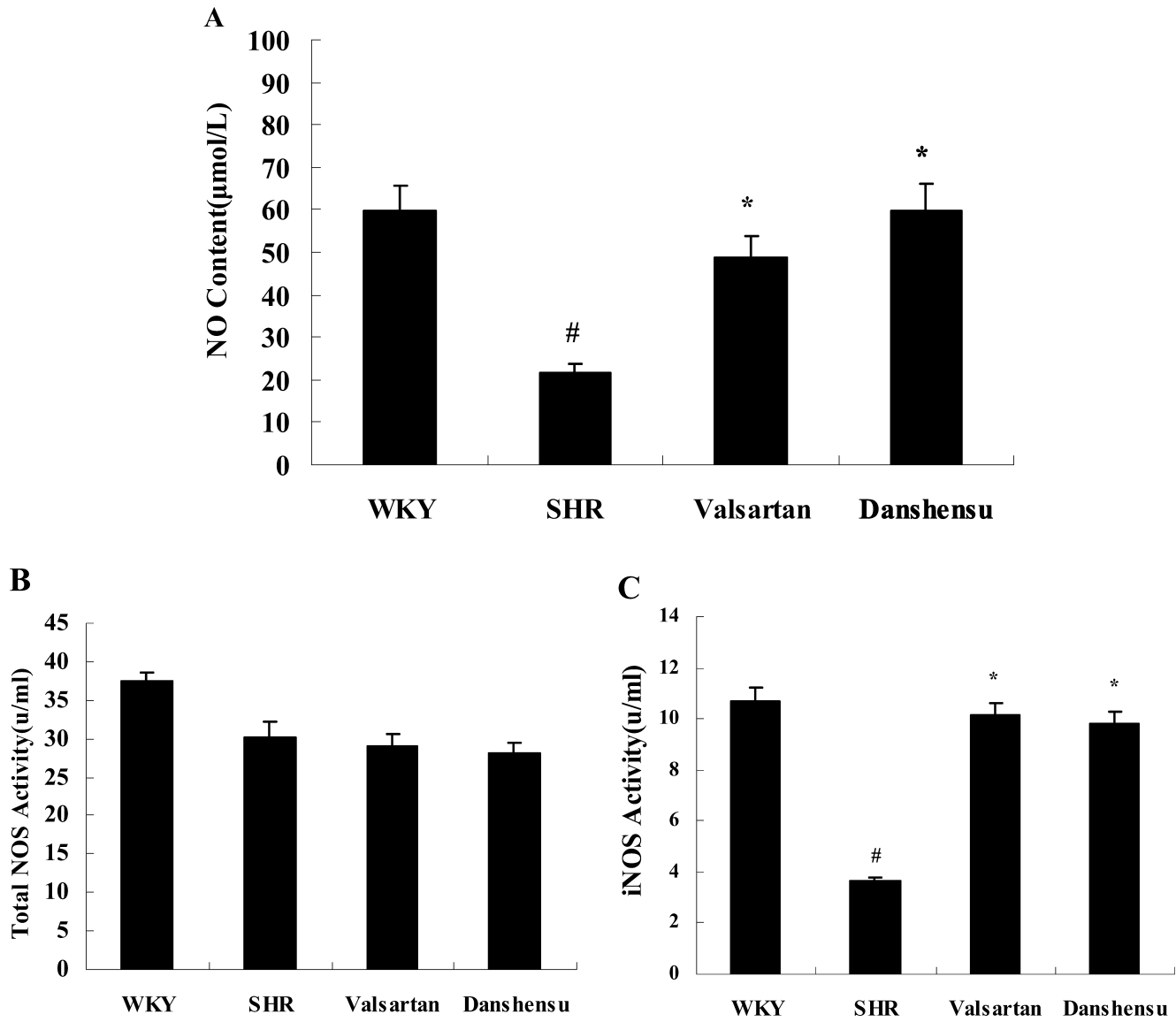

Fig. 2. Effect of Danshensu on NO Content and NOS Activity in Blood Serum

At sacrifice, blood was collected from the abdominal aorta. (A) NO content was determined by the method of nitric acid disoxidation technique. (B, C) NOS (total and iNOS) activities were determined as described in Materials and Methods. A unit of enzyme activity (u) was 1 nmol production of NO in 1 ml serum per minute. Data are presented as mean \pm S.D. ( $n=$ at least 6 rats in each group). Both Danshensu-treated and Valsartan-treated groups were administered at a dose of $10 \mathrm{mg} / \mathrm{kg}$ daily for 6 weeks. $\# p<0.05$ versus WKY. $* p<0.05$ versus SHR.

cantly reduced and MWC was significantly increased in the Danshensu group and the Valsartan group (all $p<0.05$; Table 2).

Effects of Danshensu on NO Content and NOS Activity in Serum Compared to the WKY group, NO content and iNOS activity were significantly reduced in the SHR group (both $p<0.05$ ). The decrease in NO content and iNOS activity were attenuated in the Danshensu and Valsartan groups (Fig. 2; all $p<0.05$ ).

Effects of Danshensu on Outward $\mathrm{K}^{+}$Currents and BKCa Currents While the cells were held at a constant $-40 \mathrm{mV}$ voltage, 500 -ms depolarizing pulses were applied to test potentials from -40 to $+60 \mathrm{mV}$ in $10-\mathrm{mV}$ increments and induce an outward current. The addition of TEA ( $2 \mathrm{~mm})$ inhibited the BKCa current (Fig. 3A). Figure 3B1 and B2 show the effect of Danshensu on the current-voltage $(I-V)$ relationship. The total $\mathrm{K}^{+}$current density and BKCa current density were both reduced in SHR cells compared to WKY cells. Danshensu treatment significantly enhanced both total $\mathrm{K}^{+}$current and BKCa current in SHR cells, when the test potential was set at $+60 \mathrm{mV}$ (Figs. 3B1-3; both $p<0.05$ ).

\section{DISCUSSION}

In the present study, we used the SHR model to evaluate anti-hypertensive and cardiovascular protective effects of
Danshensu. Some reports have documented that Danshensu has no significant effect on normal animal blood pressure. ${ }^{15,16)}$ Our previous study has shown that systolic blood pressure, diastolic blood pressure, and mean arterial blood pressure were $110 \pm 1,86 \pm 10,94 \pm 7 \mathrm{mmHg}$, respectively, in control male Sprague-Dawley rats, $180-250 \mathrm{~g}$ and were $112 \pm 2,79 \pm 3,94 \pm 2 \mathrm{mmHg}$, respectively, in Danshensu $(10 \mathrm{mg} / \mathrm{kg} /$ day, i.p. for $4 \mathrm{~d})$ treated rats $(p=0.33,0.44,0.93$, respectively). At a daily dose of $10 \mathrm{mg} / \mathrm{kg}$ given for 6 weeks, Danshensu normalized blood pressure and prevented the cardiac hypertrophic response. Furthermore, Danshensu showed protection against ischemia/reperfusion induced arrhythmias, as evidenced by reducing arrhythmia scores and the incidence of malignant arrhythmias. Combined, these data reveal novel cardioprotective effects of Danshensu against hypertension-induced cardiovascular remodeling.

Hypertension, defined as an elevation of systolic or diastolic blood pressure over 140 or $90 \mathrm{mmHg}$, respectively, is the most common cardiovascular disease. ${ }^{17)}$ Primary hypertension is no longer considered simply as a state of elevated blood pressure, but is rather a complex constellation of multiple abnormalities that stimulate vascular and cardiac remodeling. The SHR is a genetic chronic hypertension model. Previous research has shown that hypertension is accompanied with the structural and functional alterations in small arteries as an adaptive response to the high blood pressure. ${ }^{18)}$ 
A

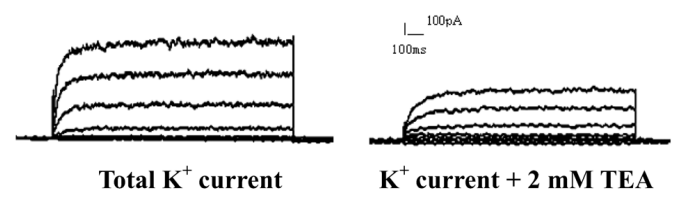

B1

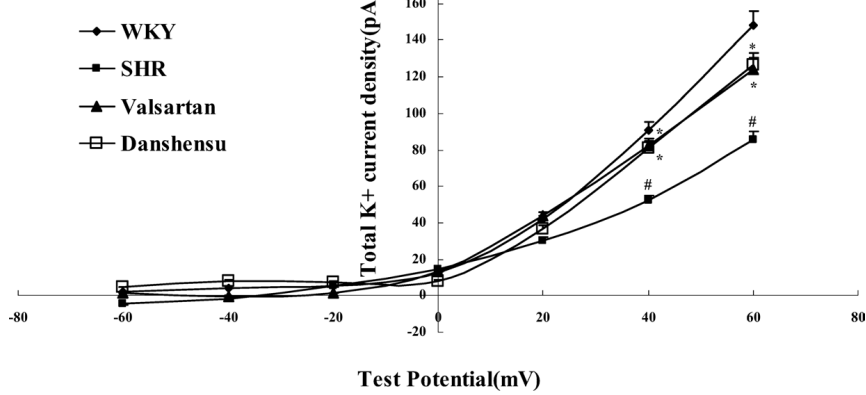

B2

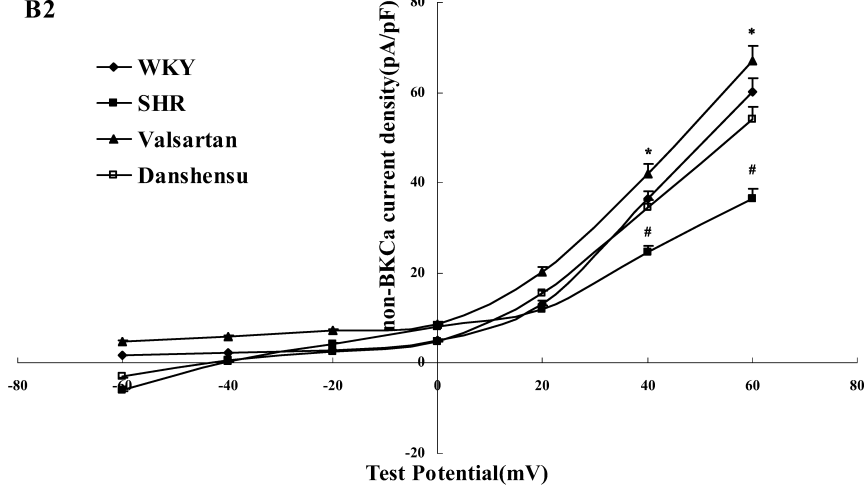

B3

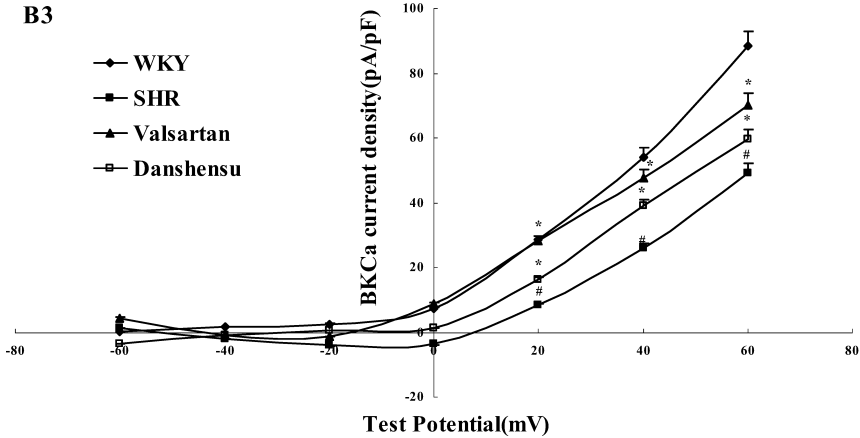

Fig. 3. Effects of Danshensu on the Total $\mathrm{K}^{+}$Current, the Non-BKCa Current, and BKCa Currents in Isolated Rat Mesenteric Vascular Smooth Muscle Cells

(A) Representative recordings of conventional whole-cell $\mathrm{K}^{+}$current elicited with $500 \mathrm{~ms}$ voltage steps from a $V_{\mathrm{h}}$ of $-40 \mathrm{mV}$ to test potentials in the range -40 to $+60 \mathrm{mV}$, measured in the absence (total $\mathrm{K}^{+}$current) and in the presence of TEA $(2 \mathrm{~mm})$. (B1) Current-voltage relationships constructed before the addition of TEA indicated the total $\mathrm{K}^{+}$current. (B2) Current-voltage relationships constructed in the presence of TEA indicated the non-BKCa current. (B3) BKCa current obtained from the total $\mathrm{K}^{+}$current minus the current stimulated by voltage in the presence of TEA. Valsartan: Valsartan treatment group, Danshensu: Danshensu treatment group. Curren density: Current/Membrane capacitance $(\mathrm{pA} / \mathrm{pF})$. Data are presented as mean \pm S.D. $(n=$ at least 6 rats in each group). $\# p<0.05 v s$. WKY. $* p<0.05 v s$. SHR

Meanwhile, SHR develops progressive hypertension ventricular hypertrophy, left ventricular fibrosis, and develop chronic failure similar to humans. ${ }^{19,20)}$

$\mathrm{K}^{+}$channels have multiple roles in vascular smooth muscle function. $\mathrm{K}^{+}$channels are required for the maintenance of vascular tone, controlling membrane potential and intracellu- lar $\mathrm{Ca}^{2+}$ signaling to regulate vasoconstriction. ${ }^{21)}$ Large conductance $\mathrm{Ca}^{2+}$-activated $\mathrm{K}^{+}$channels respond to changes in intracellular $\mathrm{Ca}^{2+}$ and regulate membrane potentials, thus playing an important role in the control of myogenic tone in vascular smooth muscle. Furthermore, voltage- and $\mathrm{Ca}^{2+}$ dependent activation of $\mathrm{BKCa}$ channels limit depolarization and vasoconstriction. Several studies have reported alterations of arterial smooth muscle potassium channel composition and BKCa currents in SHR using patch clamp technique, and obtained varying results depending on the experimental and tissue type used. There remains considerable conflict and disparity in interpretations of results, with respect to the changes and regulation of $\mathrm{K}^{+}$currents in the SHR model. ${ }^{22}$ Dr. Lam and colleagues reported that Salvianolic acid B, one of the major hydrophilic phenolics found in Salvia miltiorrhiza, elicited a concentration-dependent activation of the $\mathrm{BKCa}$ channels in porcine coronary artery smooth muscle cells. Other chemical constituents of Danshen, including Danshensu, failed to alter the gating properties of BKCa channels. ${ }^{23)}$ In this paper, we compared $\mathrm{K}^{+}$currents in smooth muscle cells from mesenteric resistance arteries of Danshensu treated-SHR. $\mathrm{K}^{+}$currents and $\mathrm{BKCa}$ channels in mesenteric vascular smooth muscle cells were down-regulated in the SHR cells, and Danshensu treatment prevented this decline. Combined with an increase in serum NO levels, these mechanisms may explain the decrease in blood pressure in the Danshensu treated SHR. The ability of eNOS to generate superoxide and reactive oxygen species at the expense of NO is referred to as eNOS uncoupling. ${ }^{24,25)}$ Our results indicate that chronic Danshensu treatment increased activation of $\mathrm{BKCa}$ by stimulating nitric oxide and this effect might contribute to the vascular protective effect. Further, NO stimulates cGMP formation. ${ }^{26)}$ The activated protein kinase $\mathrm{G}$ can close L-type voltage-dependent $\mathrm{Ca}^{2+}$ channels either by membrane hyperpolarization caused by the stimulation of $\mathrm{Ca}^{2+}$-dependent $\mathrm{K}^{+}$channels ${ }^{27)}$ or by a direct effect on L-type voltage-dependent $\mathrm{Ca}^{2+}$ channels. ${ }^{28)}$ In response to various neurohumoral stimuli, endothelial cells release nitric oxide and produce hyperpolarization of the underlying vascular smooth muscle cells. ${ }^{29,30)}$ NO stimulates smooth muscle soluble guanylyl cyclase increases cGMP and can activate ATP-sensitive, BKCa or Kv channels. ${ }^{31)}$ The increased NO levels not only can stimulate vasorelaxation, but could also exert an anti-proliferative effect on smooth muscle cells. ${ }^{32)}$ Thus NO release could be involved in the mechanism of the effects of Danshensu on vascular protection in the aorta.

In summary, Danshensu treatment in this study decreased blood pressure in the SHR model. Moreover, Danshensu significantly inhibited arrhythmias induced by ischemia reperfusion, and increased NO release. Therefore, Danshensu exerted effects on several pharmacological targets in hypertension. The inhibition potency was similar to that seen with Valsartan. Therefore, we suggest that Danshensu could be widely used in preventing the development of hypertension.

Acknowledgement This study was supported by the National Natural Science Foundation of China (No. 30772609). 


\section{REFERENCES}

1) Chen J. D., Tao J., Zhang R. J., Xu Y. H., Soong T. W., Li S. N., Peptides, 30, 1117-1123 (2009).

2) Oh K. S., Han W., Wang M. H., Lee B. H., Biol. Pharm. Bull., 30, 1278 -1283 (2007).

3) Ji K. T., Chai J. D., Xing C., Jan J. L., Yang P. L., Tang J. F., J. Zhejiang Univ. Sci. B, 11, 618-626 (2010).

4) Kim S. K., Jung K. H., Lee B. C., Biol. Pharm. Bull., 32, 220-224 (2009).

5) Zhao N., Liu Y. Y., Wang F., Hu B. H., Sun K., Chang X., Pan C. S., Fan J. Y., Wei X. H., Li X., Wang C. S., Guo Z. X., Han J. Y., Am. J. Physiol. Heart Circ. Physiol., 298, H1166-H1176 (2010).

6) Wang C. Y., Ma F. L., Liu J. T., Tian J. W., Fu F. H., Biol. Pharm. Bull., 30, 44-47 (2007).

7) Le X. Y., Chen C. L., Ma L., Zhao N., Tang Y. Q., Liu X. Q., Chin. J. Nat. Med., 6, 461-465 (2008).

8) Bao J., Ma L., Le X.Y., Chen C.L., Tang Y.Q., Acta Chinese Medicine and Pharmacology, 37, 9-11 (2009).

9) Tang Y. Q., Wang M. H., Le X. Y., Meng J. N., Huang L., Yu P., Chen J., Wu P., Phytomedicine (2011), in press.

10) Köhler R., Eur. J. Phys., 460, 343-351 (2010).

11) Cogolludo A., Frazziano G., Briones A. M., Cobeño L., Moreno L., Lodi F., Salaices M., Tamargo J., Perez-Vizcaino F., Cardiovasc. Res., 73, $424-431$ (2007).

12) Köhler R., Kaistha B. P., Wulff H., Expert Opin. Ther. Targets, 14, $143-155$ (2010)

13) Saponara S., Testai L., Iozzi D., Martinotti E., Martelli A., Chericoni S., Sgaragli G., Fusi F., Calderone V., Br. J. Pharmacol., 149, 10131021 (2006).

14) Unemoto T., Matsushita M., Tamura K., Tanaka Y., Koike K., Kogo H., Biol. Pharm. Bull., 30, 1477-1480 (2007).

15) Zhou X. M., Yang W.B., Chai Q.Y., Shanxi Med. J., December, 33, $1028-1030$ (2004).

16) Tai M. H., Liu L. M., Ma R. Q., Li X., Pang J. X., Xu J. P., J. First Mil.
Med. Univ., 25, 335-338 (2005)

17) Sarafidis P. A., Bakris G. L., J. Am. Coll. Cardiol., 52, 1749-1757 (2008).

18) Steyner F. C., Bruno A. R., Catherine C., Isac A. M., Mauro M. T., Miguel J. L., Virgínia S. L., Br. J. Pharmacol., 133, 849-858 (2001).

19) Hojná S., Kunes J., Zicha J., Physiol. Res., 59, 997-1009 (2010).

20) Chan V., Fenning A., Levick S. P., Loch D., Chunduri P., Lyer A., Teo Y. L., Hoey A., Wilson K., Burstow D., Brown L., J. Cardiovasc. Pharmacol. [EPub Ahead of Print].

21) Ko E. A., Park W. S., Firth A. L., Kim N., Yuan J. X., Han J., Prog Biophys. Mol. Biol., 103, 95-101 (2010).

22) Zhang Y., Gao Y. J., Zuo J., Lee R. M., Janssen L. J., Eur. J. Pharmacol., 514, 111-119(2005).

23) Lam F. F., Seto S. W., Kwan Y. W., Yeung J. H., Chan P., Eur. J. Pharmacol., 546, 28-35 (2006).

24) Li H., Witte K., August M., Brausch I., Gödtel-Armbrust U., Habermeier A., Closs E. I., Oelze M., Münzel T., Förstermann U., J. Am. Coll. Cardiol., 47, 2536-2544 (2006).

25) Li H., Wallerath T., Münzel T., Förstermann U., Biochemistry, 7, 149 164 (2002).

26) Lísková S., Petrová M., Karen P., Kunes J., Zicha J., Journal of the American Society of Hypertension, 4, 128 -134 (2010).

27) Jonathan L., Matthias E. W., Joseph E. B., Mark T. N., Physiology (Bethesda), 21, 69-79 (2006).

28) Yao X. Q., Huang Y., Trends Pharmacol. Sci., 24, 263-266 (2003).

29) Briones A. M., Padilha A. S., Cogolludo A. L., Alonso M. J., Vassallo D. V., Pérez-Vizcaino F., Salaices M., J. Hypertens., 27, 83-91 (2009).

30) Félétou M., Vanhoutte P. M., Arterioscler. Thromb. Vasc. Biol., 26, 1215-1225 (2006)

31) Waldron G. J., Cole W. C., Clin. Exp. Pharmacol. Physiol., 26, 180184 (1999).

32) Zhang G. S., Wang R. J., Zhang H. N., Zhang G. P., Luo M. S., Luo J. D., Biol. Pharm. Bull., 33, 427-431 (2010). 